\title{
ALGEBRAIC STRUCTURE OF THE MANIFOLD OF SOLUTIONS OF THE $N$-BODY PROBLEM
}

\author{
LAWRENCE GOLDMAN
}

\begin{abstract}
The Theorem of Ritt on the decomposition of the perfect differential ideal generated by a single irreducible differential polynomial is, here, generalized to system of polynomials satisfying certain conditions. We use these results to prove that all solutions of the $N$-body problem, excepting the solutions for which one or more of the $r_{i j}$ (the distance between the masses $M_{i}$, $M_{j}$ ) is zero, belong to one irreducible manifold.
\end{abstract}

Introduction. In [3, p. 22] Ritt proved that the manifold of zero's of a system of differential polynomials is a finite union of irreducible manifolds. If the system consists of a single irreducible differential polynomial $F$, then the manifold of $F$ breaks up into an irreducible general manifold and a certain number, possibly zero, of singular manifolds. In this paper the latter result is generalized to systems of polynomials: $F=F_{1}, \cdots, F_{n}, n \geqq 1$ satisfying certain conditions. These conditions are almost always satisfied in problems of particle mechanics. When we apply these results to the $N$-body problem, we prove that all solutions of the $N$-body problem, excepting those for which one or more of the $r_{i j}$ (the distance between the point masses $M_{i}, M_{j}$ ) is zero, belong to one irreducible manifold (the general manifold). The solutions of the $N$-body problem for which one or more of the $r_{i j}$ is zero constitute the singular manifolds. In particular, the equilateral triangle, isosceles triangle, straight line solutions of the three-body problem belong to the general manifold. However, this paper still does not answer the question whether any singular solution belongs to the general manifold.

For the applications to mechanics only Theorem 1 and Corollary 1 is needed and the number of variables can be assumed to be the same as the number of equations. In order to obtain a proper generalization of Ritt's Theorem on the decomposition of a single differential polynomial in an arbitrary number of variables we have proven our results for the case where the number of variables is $\geqq$ the number of equations, and extended these results to systems of greater generality than that required for problems in particle mechanics.

Received by the editors July 23, 1969.

AMS Subject Classifications. Primary 1280, 8520.

Key Words and Phrases. Manifold of systems, decomposition of radical ideals, decomposition of perfect differential ideals, Jacobian, $N$-body problem, three-body problem. 
Notation. To avoid repetition we shall adhere to the following: $K$ is a differential field of characteristic zero (i.e. a field with a given derivation; in our application to mechanics the derivation is the derivative with respect to the time $t$ )

$$
K\{X\}=K\left\{X_{1}, \cdots, X_{n}, X_{n+1}, \cdots, X_{n+m}\right\}
$$

is the ring of differential polynomials in the $X$ 's and all their derivatives with coefficients in $K$.

$F$ is a set of $n$ differential polynomials $F_{1}, \cdots, F_{n} \in K\{X\}$. The max order of $X_{i}$ in the system $F$ is $r_{i}, i=1, \cdots, n+m$

$$
\begin{gathered}
J=\operatorname{det}\left(\partial F_{i} / \partial X_{j}^{\left(r_{j}\right)}\right) ; i, j=1, \cdots, n, \\
R=K\left[\left(X_{i}^{(j)}\right)\left(0 \leqq j \leqq r_{i} \text { if } 1 \leqq i \leqq n ; 0 \leqq j<\infty \text { if } n<i \leqq n+m\right)\right] .
\end{gathered}
$$

$R\left\{F^{\prime}\right\}$ is the ring of polynomials in $F_{1}^{\prime}, \cdots, F_{n}^{\prime}$ (' denotes derivatives) and all their derivatives with coefficients in $R$.

$$
\tilde{R}=K\left[\left(X_{i}^{(j)}\right)\left(1 \leqq i \leqq n+m ; 0 \leqq j \leqq r_{i}\right)\right] .
$$

Note that $F \subset \widetilde{R} \subset R, J \in \widetilde{R}$. $J$ and $R$ depend on the ordering of the $X$ 's, while $F$ and $\widetilde{R}$ do not.

$(F), \sqrt{ } F$, is the ideal, radical ideal, respectively in the ring $R$ or $\widetilde{R}$ generated by $F$.

$[F],\{F\}$, is the differential ideal, perfect (radical) differential ideal, respectively in the differential ring $K\{X\}$.

If $A$ is a radical ideal of a ring $U$ and $v \in U$, then $A: v$ is the radical ideal consisting of all $u \in U$ such that $v u \in A$.

$\nu$ will always denote a nonnegative integer.

$K\langle f\rangle$ denotes the differential field extension of $K$ by $f$.

Lemma 1. For any $Q \in K\{X\}$ there exists $\nu$ such that $J^{\nu} Q \in R\left\{F^{\prime}\right\}$. If $J \neq 0$ then the elements $\left(F_{i}^{(D)}\right)(1 \leqq i \leqq n ; 1 \leqq j<\infty)$ are algebraically independent over $R$.

Proof. Let $J \neq 0$, let $T\left(Y^{\prime}\right) \in R\left[\left(Y_{i}^{(\jmath)}\right)(1 \leqq i \leqq n ; 1 \leqq j<\infty)\right]$ and let $T\left(F^{\prime}\right)=0$. Let $p$ be the highest order derivative of the $Y_{i}$ occurring in $T$. Then

$$
\begin{aligned}
0=\partial T / \partial X_{k}^{\left(r_{k}+p\right)} & =\sum_{i=1}^{n}\left(\partial T / \partial F_{i}^{(p)}\right)\left(\partial F_{i}^{(p)} / \partial X_{k}^{\left(r_{k}+p\right)}\right) \\
& =\sum_{i=1}^{n}\left(\partial T / \partial F_{i}^{(p)}\right)\left(\partial F_{i} / \partial X_{k}^{\left(r_{k}\right)}\right) .
\end{aligned}
$$


This is a system of $n$ linear homogeneous equations with determinant $J \neq 0$, so that $\partial T / \partial F_{i}^{(p)}=0, i=1, \cdots, n$.

To show existence, we note that the set of $Q \in K\{X\}$ for which Lemma 1 holds is a ring which contains $R$. It suffices, therefore, to prove existence for $X_{i}^{(j)} ; 1 \leqq i \leqq n, r_{i}<j<\infty$. Assume inductively that the lemma holds for $X_{i}^{(j)}, 1 \leqq i \leqq n ; r_{i}<j<p$. Consider the system of $n$ equations

$$
\begin{aligned}
F_{i}^{(p)} & =\sum_{j=1}^{n}\left(\partial F_{i}^{(p-1)} / \partial X_{j}^{\left(r_{j}+p-1\right)}\right) X_{j}^{\left(r_{j}+p\right)}+A_{i} \\
& =\sum_{j=1}^{n}\left(\partial F_{i} / \partial X_{j}^{\left(r_{j}\right)}\right) X_{j}^{\left(r_{j}+p\right)}+A_{i}, \quad i=1, \cdots, n
\end{aligned}
$$

$A_{i} \in K\left[\left(X_{\boldsymbol{k}}^{(\Uparrow)}\right) \quad\left(1 \leqq k \leqq n+m ; 0 \leqq j<r_{k}+p\right)\right]$. By our induction assumption there exists $\nu$ such that $J^{\nu} A_{i} \in R\left\{F^{\prime}\right\}, i=1, \cdots, n$. Since the determinant of this system is $J$, we have $J^{v+1} X_{j}^{r_{j+p}} \in R\left\{F^{\prime}\right\}$.

Corollary. $\{F\}: J \cap R=\sqrt{ } F: J$.

Proof. Clearly $\sqrt{ } F: J \subset\{F\}: J \cap R$. Let $Q \in\{F\}: J \cap R$, then $(J Q)^{\nu} \in[F] \cap R$. By the algebraic independence of $\left(F_{i}^{(j)}\right)(1 \leqq k \leqq n$; $1 \leqq j<\infty)$ over $R$, we have $(J Q)^{\nu} \in(F)$ so that $J Q \in \sqrt{ } F$ and $Q \in \sqrt{ } F: J$.

TheOREM 1. Let $\odot$ be a prime ideal of $R$, let $F \subset \odot$, let $J \notin \odot$ and let $\{\odot\}: J \cap R=\odot$. Then $\{\odot\}: J$ is a prime differential ideal of $K\{X\}$ and is a minimal differential prime divisor of $\{\rho\}$.

Proof. Let $P Q \in\{\odot\}: J ; P, Q \in K\{X\}$. By Lemma 1 there exists $\nu$ such that $J^{\nu} P \equiv \bar{P}\left[F^{\prime}\right], J^{\nu} Q \equiv \bar{Q}\left[F^{\prime}\right] ; \bar{P}, \bar{Q} \in R$. Thus $\overline{P Q} \in\{\odot\}: J$ $\cap R=\odot$. Since $\rho$ is prime, one of $\bar{P}, \bar{Q}$, say $\bar{P} \in \mathcal{P}$ and $P \in\{\rho\}: J$. Hence $\{\rho\}: J$ is a prime differential ideal of $K\{X\}$. Also, any prime differential ideal containing $\{\rho\}$ and not containing $J$ must contain $\{\odot\}: J$. Hence $\{\odot\}: J$ is a minimal differential prime divisor of $\{\rho\}$.

Corollary 1. Let $J \notin \sqrt{ } F$ and let $\sqrt{ } F: J$ be a prime polynomial ideal of $R$. Then $\{F\}: J$ is a prime differential ideal of $K\{X\}$.

Proof. Let $P=\sqrt{ } F: J$ then $\{\odot\}: J=\{\sqrt{ } F: J\}: J \subset\{\{F\}: J\}: J$ $=\{F\}: J$. Now, $\{F\}: J \cap R=\sqrt{ } F: J$ (corollary of Lemma 1) and by Theorem $1\{F\}: J$ is a prime differential ideal of $K\{X\}$.

Corollary 2. Let $(F)$ be a prime ideal of $R$ and let $J \notin(F)$. Then the differential dimension of $\{F\}: J$ is $m$. 
Proof. Let $f=\left(f_{1}, \cdots, f_{n+m}\right)$ be a generic zero of $\{F\}: J$. Since $J \notin\{F\}: J, J$ does not vanish at $X=f$. Thus

$$
\begin{aligned}
f_{i}^{\left(r_{i}+1\right)} \in K\left\langle f_{n+1}, \cdots, f_{n+m}\right\rangle\left(\left(f_{k}^{(j)}\right) \quad(1 \leqq k \leqq n ; 0 \leqq\right. & \left.\left.j \leqq r_{k}\right)\right), \\
i & =1, \cdots, n .
\end{aligned}
$$

Hence differential dimension of $\{F\}: J$ is $\leqq m$. Let $T \in K\left\{X_{n+1}, \cdots\right.$, $\left.X_{n+m}\right\}$ vanish at $X=f$. Then $T \in\{F\}: J \cap R=\sqrt{ } F: J=(F)$. Let $s_{i}=\max \left(\right.$ order of $T$ in $\left.X_{i}, r_{i}\right), i=1, \cdots, n+m$, then $\rho$, the polynomial ideal generated by $F$ in the polynomial ring $K\left[\left(X_{i}^{(j)}\right)\left(1 \leqq i \leqq n+m ; 0 \leqq j \leqq s_{i}\right)\right]$ is a prime ideal with generic zero $\left(f_{i}^{(j)}\right), 1 \leqq i \leqq n+m ; 0 \leqq j \leqq s_{i}$. Now rank of $\left.\left(\partial F_{i} / \partial X_{j}^{\left(s_{j}\right)}\right)\right|_{X=f}$ is $n$. Hence transcendence degree of $L=K\left(\left(f_{i}^{(j)}\right)\left(1 \leqq i \leqq n+m ; 0 \leqq j \leqq s_{i}\right)\right)$ over $K$ is $\sum_{i=1}^{n+m}\left(s_{i}+1\right)-n$ and $\left(f_{i}^{(j)}\right), 1 \leqq i \leqq n+m, 0 \leqq j<r_{i}$ if $1 \leqq i \leqq n$, $0 \leqq j \leqq s_{i}$ if $n<i \leqq n+m$, is a transcendence base for $L$ over $K$ [1, p. 84]. Hence $T=0$ and differential dimension of $\{F\}: J$ is $m$.

REMARK. When $n=1, F=F_{1}, F$ irreducible over $K$, then $(F)=\sqrt{ } F$ $=\sqrt{ } F: J(J$ is the separant of $F)$ is a prime polynomial ideal of $R$. We thus, obtain Ritt's Theorem on the decomposition of $\{F\}$ [3, p. 31]. For this case using the fact that $\partial F / \partial X_{i}^{\left(r_{i}\right)} \notin\{F\}: J$, $i=1, \cdots, m+1$, Ritt obtained the additional result that $\{F\}: J$ is independent of the ordering of the $X_{i}$. Thus one can speak of the manifold of $\{F\}: J$ as the general manifold and of the other irreducible components of $\{F\}$ as the singular manifold of $\{F\}$. We can extend these notions to the case where $n>1$ if we assume the following:

(1) The radical ideal $\odot$ generated by $F$ in

$$
\widetilde{R}=K\left[\left(X_{i}^{(j)}\right)\left(1 \leqq i \leqq n+m ; 0 \leqq j \leqq r_{i}\right)\right]
$$

is a prime polynomial ideal.

(2) Let $J$ be the Jacobian of $F$ with respect to the highest order derivatives of any $n$ of the $n+m$ indeterminates $X_{i}$. Then $J \notin \odot$ (that is, $J \notin \odot$ for every ordering of the $X_{i}$ ).

For such a system $F$ we can again speak of the manifold of $\{F\}: J$ as the general manifold of $\{F\}$, and of the other irreducible components of the manifold of $\{F\}$ as the singular manifolds of $\{F\}$. For clearly, $\sqrt{ } F=R \odot$ is a prime ideal of $R \supset \widetilde{R}$, so that, by Corollary 1 of Theorem $1,\{F\}: J$ is a prime differential ideal of $K\{X\}$; if the Jacobian $J^{*}$ for some reordering of the $X_{i}$ were in $\{F\}: J$ then we would have

$$
J^{*} \in\{F\}: J \cap R \cap \tilde{R}=\sqrt{ } F \cap \tilde{R}=\odot,
$$

contrary to assumption (2), above. 
TheOREm 2. Let $\odot$ be a prime ideal of $R$ such that $F \subset \odot$ and $J \notin \odot$. Let $X=f$ be a zero of $\{\odot\}$ such that $f$ is a generic zero of $P$. Then $\{\odot\}: J$ is a prime differential ideal of $K\{X\}$ with generic zero $f$.

Proof. Let $Q \in\{\odot\}: J \cap R$. Since $J(f) \neq 0, Q(f)=0$ and $Q \in \odot$. Thus $\{\odot\}: J \cap R=\odot$, by Theorem $1,\{\odot\}: J$ is a prime differential ideal. Let $g$ be any zero of $\{\rho\}$ such that $J$ does not vanish at $g$, then $f \rightarrow g$ is a differential specialization over $K$. For, let $Q \in K\{X\}$ and let $Q(f)=0$, then there exists $\nu$ such that $J^{\nu} Q \equiv \bar{Q}\left[F^{\prime}\right], \bar{Q} \in R$ (by Lemma 1). Hence $\bar{Q}(f)=0$ and $\bar{Q} \in \mathcal{P}$. Thus $\bar{Q}(g)=0$ and since $J(g) \neq 0, Q(g)=0$. Therefore $f \rightarrow g$ is a differential specialization over $K$ and $f$ is generic zero of $\{\rho\}: J$.

TheOREM 3. Let $\boldsymbol{P}_{1}, \cdots, \odot_{s}$ be a complete set of irredundant minimal prime ideal divisors of $F$ not containing $J$ and let $\left\{\odot_{i}\right\}: J \cap R=\odot_{i}$; $i=1, \cdots, s$. Then $\left\{\mathcal{P}_{1}\right\}: J, \cdots,\left\{\mathcal{P}_{s}\right\}: J$ is a complete set of irredundant minimal prime differential ideal divisors of $F$ not containing $J$.

Proof. Since $\left\{\odot_{i}\right\}: J \cap R=\odot_{i}, J \notin \odot_{i}$ and by Theorem $1,\left\{\odot_{i}\right\}: J$ is a prime differential ideal of $K\{X\}$. Let $D$ be any prime differential ideal divisor of $F$ not containing $J$, then $D \cap R$ is a prime ideal divisor of $F$ not containing $J$. Thus $D \cap R$ contains some $\mathcal{P}_{i}$ and $D \supseteq\{D \cap R\}: J \supseteq\left\{P_{i}\right\}: J$. Hence if $D$ is a minimal prime differential divisor of $F$ then $D=\left\{Q_{i}\right\}: J$. To show irredundance, let $\left\{\odot_{i}\right\}: J$ $\supseteq\left\{\odot_{j}\right\}: J$, then $\left\{\odot_{i}\right\}: J \cap R \supseteq\left\{\odot_{j}\right\}: J \cap R$ and $\odot_{i} \supseteq \odot_{j}$. Hence $i=j$ and $\left\{\mathbb{P}_{i}\right\}: J, \cdots,\left\{\mathbb{P}_{s}\right\}: J$ form a complete set of irredundant minimal prime differential ideal divisors of $F$.

The $N$-body problem. The cartesian coordinates $\left(x_{i}, y_{i}, z_{i}\right)$, $i=1, \cdots, N$ of $N$ point masses $M_{i}$, subject only to their mutual gravitational forces satisfy the following system of equations:

$$
\begin{gathered}
x_{i}^{\prime \prime}-\sum_{j \neq i} M_{j}\left(x_{j}-x_{i}\right) / r_{i j}^{3}=0, \\
y_{i}^{\prime \prime}-\sum_{j \neq i} M_{j}\left(y_{j}-y_{i}\right) / r_{i j}^{3}=0, \quad i=1, \cdots, N, \\
z_{i}^{\prime \prime}-\sum_{j \neq i} M_{j}\left(z_{j}-z_{i}\right) / r_{i j}^{3}=0, \\
r_{i j}^{2}-\left(x_{i}-x_{j}\right)^{2}-\left(y_{i}-y_{j}\right)^{2}-\left(z_{i}-z_{j}\right)^{2}=0, \quad 1 \leqq i<j \leqq N,
\end{gathered}
$$

where $r_{i j}$ denotes $r_{j i}(1 \leqq j<i \leqq N)$.

The left-hand side of this system of equations when cleared of fractions is a system $F$ of $n=3 N+N(N-1) / 2$ differential polynomials in $n$ variables. $F$ belongs to the differential ring $K\{X\}$ where 


$$
K\{X\}=K\left\{x, y, z,\left(r_{i j}\right)(1 \leqq i \leqq N ; 1 \leqq j \leqq N ; i \neq j)\right\}
$$

(we write $x$ for $x_{1}, \cdots, x_{N}$ etc.) and $K$ is the field of constants $Q\left(M_{1}, \cdots, M_{n}\right), Q=$ rationals. In accordance with our notation above, $R=K\left[x, y, z, x^{\prime}, y^{\prime}, z^{\prime}, x^{\prime \prime}, y^{\prime \prime}, z^{\prime \prime},\left(r_{i j}\right)\right] ; J$ is a power of $\prod r_{i j}$. This system $F$, when we look at it as a system of polynomials in the ring $R$, is a characteristic set (i.e. is in triangular form) with a parametric set consisting of the $6 N$ elements $x, x^{\prime}, y, y^{\prime}, z, z^{\prime}$. It is not difficult to show that this characteristic set satisfies the irreducibility conditions of Ritt [3, p. 89]. Thus $F$ is a characteristic set of a prime polynomial ideal. Also, $J$ is a power of the product of the initials of $F$, so that $(F): J^{\infty}$ (consisting of all $Q \in R$ such that $J^{\nu} Q \in(F), \nu$ depending on $Q)$ is a prime polynomial ideal [3, p. 97]. Since $(F): J^{\infty}=\sqrt{ } F: J$, it follows from Corollary 1 , Theorem 1 that $\{F\}: J$ is a prime differential ideal in the differential ring $K\left\{x, y, z,\left(r_{i j}\right)\right\}$ and $\{F\}=\{F\}: J \cap\{F, J\}$. Thus any zero of $F$ for which $\prod r_{i j} \neq 0$, is a point of the general manifold of $\{F\}$. In particular the equilateral triangle solution of Lagrange and the isosceles triangle solution of the three body problem are points of the general manifold. This is not, entirely, without analytic significance. For, some doubt has been raised about the validity of the practice of astronomers of obtaining periodic solutions of the three body problem by perturbing the initial conditions of the equilateral triangle solution $[2, \S \mathrm{s}, \mathrm{pp} .75-78]$. This practice, clearly, would have been completely without merit if the equilateral triangle solution had been in a separate manifold. While our results do not prove the existence of periodic solutions, they do prove that the equilateral triangle solution is a limit of nonequilateral triangle solutions (possibly complex solutions) [3, p. 123].

\section{REFERENCES}

1. W. V. D. Hodge and D. Pedoe, Methods of algebraic geometry, Vol. II, Cambridge Univ. Press, Cambridge, 1952. MR 13, 972.

2. E. Leimanis, Some recent advances in the dynamics of rigid bodies and celestial mechanics. Dynamics and nonlinear mechanics, Surveys in Appl. Math., vol. 2, Wiley, New York and Chapman \& Hall, London, 1958. MR 20 \#2877.

3. J. F. Ritt, Differential algebra, Amer. Math. Soc. Colloq. Publ., vol. 33, Amer. Math. Soc., Providence, R. I., 1950. MR 12, 7.

Stevens Institute of Technology, Hoboken, New Jersey 07030 